\title{
ASPECTS OF IMPATIENCE IN A FINITE BUFFER QUEUE
}

\author{
Medhi Pallabi ${ }^{1}$ And Amit Choudhury ${ }^{1}$
}

\begin{abstract}
In a multi server queuing system, buffer size is often larger than the number of servers. This necessitates queuing and waiting for some customers. Customers become impatient while waiting for service. Additionally, they may also become impatient if service is not offered at the desired rate. This paper analyses a finite buffer multi server queuing system with the additional restriction that customers may balk as well as renege. Closed form expressions of a number of performance measures are presented. A design problem is discussed to demonstrate the results derived.
\end{abstract}

Keywords. Balking, finite Buffer, impatience, reneging, queuing.

Mathematics Subject Classification. 60K25, 68M20, $90 \mathrm{~B} 22$.

\section{INTRODUCTION}

Customers are often required to queue up and wait whenever they desire service. The prospect of waiting induces impatience on all customers. An arriving customer faced with the prospect of waiting may decide against joining the queue and leaving it for good. In queuing parlance, this is known as balking. Haight [27] has provided a rationale that might influence a person to balk. It relates to the perception of the importance of being served which induces an opinion somewhere in between urgency, so that a queue of certain length will not be joined, to indifference where a non-zero queue is also joined. Even if a customer joins the queue, it is logical to expect that the customer has a patience time beyond which he is not willing to wait. If service is not over within this patience time, the customer will leave the system also for good. The phenomenon of a customer joining the queuing system and leaving it before completing service is known as reneging.

Received December 11, 2011. Accepted June 15, 2012.

1 Department of statistics, Guwahati university, Guwahati-781014 Assam, India. pmedhi16@gmail.com 
Reneging can be of two types: reneging till beginning of service (henceforth referred to as R_BOS) and reneging till end of service (henceforth referred to as R_EOS). R_BOS can be observed in queuing systems where a customer can renege only as long as he is in queue. Once he begins receiving service, he cannot renege. A common example is the barbershop. A customer can renege while he is waiting in queue. However once service starts i.e. hair cut begins, the customer does not leave till hair cutting is over. On the other hand, R_EOS can be observed in queuing systems where a customer can renege not only while waiting in queue but also while receiving service. A serious patient in operation theatre (O.T.) is one such example because in an O.T., a patient may expire even when the service i.e. treatment is going on. Both these types of reneging are treated separately in this paper.

It is not difficult to see that the implication of impatience is loss of business not to mention the loss of good will. The management of any queuing system would therefore be interested in having quantifiable measures which can gauge the extent of business lost. Even though balking and reneging have been discussed in the literature of queuing theory, useable closed form expressions are not always available. This paper is an attempt in this direction.

In this paper, we analyze a multiserver Markovian queuing system with limited waiting space under the assumption that customers may balk as well renege. In particular, we shall assume that there are c servers with system capacity restricted to $k(k \geqslant c)$. The arrival process will be assumed to follow Poisson law with parameter $\lambda$. Servers work independent of each other and service time of each server follows Poisson law with parameter $\mu$. We assume that balking is state dependent. Specifically, it will be assumed that if the customer on arrival observes the system to be in state ' $n$ ', the probability that he will balk is ' $(n-c+1) /(k-c+1)$ ', $n=c$, $c+1, \ldots, k$. With this set up, the finite buffer restriction can also be seen as the state from which customer balks with probability $1\{=(k-c+1) /(k-c+1)\}$. There is no balking from an empty queue. All customers who join the queuing system will be assumed to be of reneging type with patience time also following Poisson law with parameter $\nu$. The patience time commences from the moment the customer joins the system. Both types of reneging R_BOS and R_EOS will be treated separately in this paper.

The subsequent sections of this paper are structured as follows. Section 2 contains a brief review of the literature. Sections 3 and 4 contains the derivation of steady state probabilities and performance measures respectively. We perform sensitivity analysis in Section 5. A numerical example is discussed in Section 6 . Concluding statements are given in Section 7. Appendix A contains some derivations.

\section{LiterATURE SURVEY}

Barrer [9] carried out one of the early work on reneging where he considered deterministic reneging with single server Markovian arrival and service rates. Customers were selected randomly for service. In his subsequents work, Barrer [10] 
also considered deterministic reneging (of both R_BOS and R_EOS type) in a multi server scenario with FCFS discipline. The general method of solution was extended to two related queuing problems. Another early work was by Haight [28]. Ancher and Gafarian [5] carried out an early work on Markovian reneging with Markovian arrival and service pattern. Ghosal [25] considered a D/G/1 model with deterministic reneging. Gavish and Schweitzer [24] considered a deterministic reneging model with the additional assumption that arrivals can be labeled by their service requirement before joining the queue and arriving customers are admitted only if their waiting plus service time do not exceed some fixed amount. Kok and Tijms [32] considered a single server queuing system where a customer becomes a lost customer when its service has not begun within a fixed time. Haghighi et al. [26] considered a Markovian multiserver queuing model with balking as well as reneging. Each customer had a balking probability which was independent of the state of the system. Reneging discipline considered by them was R_BOS. Liu et al. [35] considered an infinite server Markovian queuing system with reneging of type R_BOS. Customers had a choice of individual service or batch service, batch service being preferred by the customer. Brandt and Brandt [13] considered a S-server system with two FCFS queues, where the arrival rates at the queues and the service may depend on number of customers ' $n$ ' being in service or in the first queue. Customers in the first queue were assumed impatient customers with deterministic reneging. Boots and Tijms [11] considered an M/M/C queue in which a customer leaves the system when its service has not begun within a fixed interval after its arrival. They have given the probabilistic proof of 'loss probability', which was expressed in a simple formula involving the waiting time probabilities in the standard $\mathrm{M} / \mathrm{M} / \mathrm{C}$ queue. Ke and Wang [31] considered the machine repair problem in which failed machines balk with probability (1-b) and renege according to a negative exponential distribution. Another work using the concepts of balking and reneging in machine interference queue has been carried out by Al-Seedy and Al-Ibraheem [3]. Bae et al. [7] considered an M/G/1 queue with deterministic reneging. They derived the complete formula of the limiting distribution of the virtual waiting time explicitly. Choi et al. [14] introduced a simple approach for the analysis of the $\mathrm{M} / \mathrm{M} / \mathrm{C}$ queue with a single class of customers and constant patience time by finding simple Markov process. Applying this approach, they analyzed the M/M/1 queue with two classes of customer in which class 1 customer have impatience of constant duration and class 2 customers have no impatience and lower priority than class 1 customers. Performance measures of both $\mathrm{M} / \mathrm{M} / \mathrm{C}$ and $\mathrm{M} / \mathrm{M} / 1$ queues were discussed. Zhang et al. [48] considered an $\mathrm{M} / \mathrm{M} / 1 / \mathrm{N}$ framework with Markovian reneging where they derived the steady state probabilities and formulated a cost model. Some performance measures were also discussed. Choudhury [16] provided a detailed and lucid derivation of the distribution of virtual waiting time in a single server Markovian queuing system under R_BOS.

El-Paoumy [20] derived the analytical solution of $\mathrm{M}^{x} / \mathrm{M} / 2 / \mathrm{N}$ queue for batch arrival system with Markovian reneging. Another paper on Markovian reneging 
was by Altman and Yechiali [2]. They derived the probability generating function of number of customers present in the system and some performance measures were discussed. Xiong et al. [44] considered a single server queue with a deterministic reneging time motivated by the timeout mechanism used in application servers in distributed computing environments. They had employed a Volterra integral equation to study the $\mathrm{M} / \mathrm{G} / 1$ queue with reneging using level crossing analysis. They derived the probability generating function of number of customers present in the system and some performance measures were discussed. Jouini et al. [30] considered two multi-class call center models with and without reneging. They assumed that customers had different priorities and the content of different types of calls was assumed as similar allowing their service times to be identical. Xiong and Altiok [43] have provided approximations for some performance measures of a multi server queue with Poisson arrivals, general service time distribution and deterministic reneging.

Altman and Yechiali [1] considered "queuing systems with server vacations where each arriving customer who finds no servers on duty activates an independent random impatience timer. If a server does not show up by the time the timer expires, the customer abandons the queue". Yechiali [45] considered a multi server Markovian queue suffering occasional disaster breakdown. During such breakdown, all customers in the system are cleared. New arrivals during the breakdown period have an exponentially distributed patience time, such that if the service is not reactivated during this patience time, the customer reneges.

Other attempts at modeling reneging phenomenon include those by Baccelli et al. [8], Martin and Artalejo [36], Shawky [39], Choi et al. [15], and Singh et al. [42], El-Sherbiny [23] and El-Paoumy and Ismail [22] etc.

An early work on balking was carried out by Haight [27]. Liu and Kulkarni [33] considered an M/PH/1 queue with work load-dependent balking. They assumed that an arriving customer joined the queue and stayed until served if and only if the system workload was less than a fixed level at the time of his arrival. They also obtained the mean and LST of the busy period in the M/PH/1 queue with workload-dependent balking as a special limiting case of this fluid model. They illustrate the results with the help of numerical examples. Liu and Kulkarni [34] also considered the virtual queuing time (vqt, also known as work-in-system, or virtual delay) process in an $\mathrm{M} / \mathrm{G} / \mathrm{s}$ queue with impatient customers. They focused on the vqt-based balking model and related it to reneging behavior of the vqt process. Jouini et al. [30] modeled a call center as an $\mathrm{M} / \mathrm{M} / \mathrm{s}+\mathrm{M}$ queue with endogenized customer reactions to announcements. They assumed that customers react by balking upon hearing the delay announcement and may subsequently renege if their realized waiting time exceeds the delay that has originally announced to them. They calculated the waiting time distribution i.e. announcement coverage and subsequent performance in terms of balking and reneging. Al-Seedy et al. [4] presented an analysis for the $\mathrm{M} / \mathrm{M} / \mathrm{c}$ queue with balking and reneging. They assumed that arriving customers balked with a fixed probability and reneged according to a negative exponential distribution. The generating function 
technique was used to obtain the transient solution of system those results in a simple differential equation. Yue et al. [47] considered an M/M/2 queuing system with balking and two heterogeneous servers, server 1 and server 2 . They assumed that customers arrived according to a Poisson process and form a single waiting line where two parallel servers provided heterogeneous exponential service on a first-come first-served basis. It is also assumed that server 1 is perfectly reliable and server 2 is subject to breakdowns. They obtained the stationary condition where the system reaches a steady state and derived the steady state probabilities in a matrix form by using matrix-geometric solution method. They produced explicit expressions of some performance measures such as the mean system size, the average balking rate and the probabilities that server 2 is in various states.

Bae and Kim [6] considered a G/M/1 queue in which the patience time of the customers is constant. The stationary distribution of the workload of the server, or the virtual waiting time was derived by the level crossing argument. Boxma et al. [12] considered an M/G/1 queue in which an arriving customer does not enter the system whenever its virtual waiting time i.e. the amount of work seen upon arrival, was larger than a certain random patience time. They determined the busy period distribution for various choices of the patience time distribution. Cochran and Brayles [19] proposed to enable strategic decision making on future Emergency department (ED) based on patient safety (rather than congestion measure). They hypothesized that the Leave without treatment (LWOT) reneging percentage is captured by the balking probability $\left(p_{k}\right)$ relationship of an $\mathrm{M} / \mathrm{M} / 1 / \mathrm{K}$ queue. They derived the form of a binomial response nonlinear weighted regression model that best fits $p_{k}$ for predicting LWOT to long term ED performance by means of Gauss-Newton linearization. Yue and Yue [46] studied a two-server Markovian network system with balking and a Bernoulli schedule under a single vacation policy server had different rates. After every service, only one server might take a vacation or continued to stay in the system where the vacation time followed an exponential distribution. They obtained the steady state condition, the stationary distribution of the number of customers in the system and the mean system size by using a matrix-geometric method. Choudhury and Medhi [17] analyzed a multiserver Markovian queuing system under the assumption that customers may balk as well as renege. Explicit closed form expressions were presented. A numerical example with design aspects was also discussed to demonstrate results derived. Choudhury and Medhi [18] also analyzed a single server finite buffer Markovian queuing model $\mathrm{M} / \mathrm{M} / 1 / \mathrm{K}$ with the additional restriction that customers may balk as well as renege. In this paper, balking along with position dependent reneging was considered. Explicit closed form expressions of a number of performance measures were presented. A typical problem was discussed to demonstrate the usefulness of results derived.

Some other papers which have considered both balking and reneging are the work by Shawky and El-Paoumy [40], El-Paoumy [20,21], El-Sherbiny [23], Shawky and El-Paoumy [41], Pazgal et al. [37]. 


\section{The System State Probabilities}

In this section, the steady state probabilities are derived by the Markov process method. We first analyze the case where customers renege only from the queue. Under R_BOS, let $p_{n}$ denote the probability that there are ' $n$ ' customers in the system. The steady state probabilities under R_BOS are

$$
\lambda p_{0}=\mu p_{1},
$$

$$
\lambda p_{n-1}+(n+1) \mu p_{n+1}=\lambda p_{n}+n \mu p_{n} ; n=1,2, \ldots, c-1
$$

$$
\begin{aligned}
\lambda\{1-(n-c) /(k-c+1)\} p_{n-1}+\{c \mu+(n-c+1) \nu\} & p_{n+1} \\
=\lambda\{1-(n-c+1) /(k-c+1)\} p_{n}+\{c \mu & +(n-c) \nu\} p_{n} \\
& n=c, c+1, \ldots, k-1
\end{aligned}
$$

$$
\lambda\{1-(k-c) /(k-c+1)\} p_{k-1}=\{c \mu+(k-c) \nu\} p_{k} .
$$

Solving recursively, we get (under R_BOS)

$$
\begin{aligned}
& p_{n}=\left\{\lambda^{n} /\left(n ! \mu^{n}\right)\right\} p_{0} ; \quad n=1,2, \ldots, c \\
& p_{n}=\left[\lambda^{n} \prod_{r=1}^{n-c}\{1-r /(k-c+1)\} /\left\{c ! \mu^{c} \prod_{r=1}^{n-c}(c \mu+r \nu)\right\}\right] p_{0} ; \\
& n=c+1, \ldots, k
\end{aligned}
$$

where $p_{0}$ is obtained from the normalizing condition $\sum_{n=0}^{k} p_{n}=1$ and is given as

$$
p_{0}=\left[\sum_{n=0}^{c} \lambda^{n} /\left(n ! \mu^{n}\right)+\sum_{n=c+1}^{k} \lambda^{n} \prod_{r=1}^{n-c}\{1-r /(k-c+1)\} /\left\{c ! \mu^{c} \prod_{r=c+1}^{n}(c \mu+r \nu)\right\}\right]^{-1} .
$$

The steady state probabilities satisfy the recurrence relation, under R_BOS

$$
\begin{array}{ll}
p_{n}=\{\lambda /(n \mu)\} p_{n-1} ; & n=1,2, \ldots, c, \\
p_{n}=\{\lambda(1-\overline{n-c / k-c+1}) /(c \mu+\overline{n-c} \nu)\} p_{n-1} ; & n=c+1, \ldots, k .
\end{array}
$$

We shall denote by $S_{\mathrm{R} \_ \text {BOS }}$ the probability that an arriving unit has to wait on arrival (under R_BOS). Then

$$
S_{\mathrm{R} \_ \text {BOS }}=\sum_{n=c}^{k} p_{n}
$$


Under R_EOS where customer may renege from the queue as well as while receiving service, let $q_{n}$ denote the probability that there are $\mathrm{n}$ customers in the system. Applying the Markov theory, we obtain the following set of steady state equations.

$$
\begin{gathered}
\lambda q_{0}=(\mu+\nu) q_{1}, \\
\lambda q_{n-1}+(n+1)(\mu+\nu) q_{n+1}=\lambda q_{n}+n(\mu+\nu) q_{n} ; \quad n=1,2, \ldots, c-1, \\
\lambda\{1-(n-c) /(k-c+1)\} q_{n-1}+\{c \mu+(n+1) \nu\} q_{n+1} \\
=\lambda\{1-(n-c+1) /(k-c+1)\} q_{n}+\{c \mu+n \nu\} q_{n} ; \\
n=c+1, \ldots, k-1 \\
\lambda\{1-(k-c) /(k-c+1)\} q_{k-1}=\{c \mu+k \nu\} q_{k} .
\end{gathered}
$$

Solving recursively, we get (under R_EOS)

$$
\begin{gathered}
q_{n}=\left[\lambda^{n} /\left\{n !(\mu+\nu)^{n}\right\}\right] q_{0} ; \quad n=1,2, \ldots, c \\
q_{n}=\left[\lambda^{n} \prod_{r=1}^{n-c}\{1-r /(k-c+1)\} /\left\{c !(\mu+\nu)^{c} \prod_{r=c+1}^{n}(c \mu+r \nu)\right\}\right] q_{0} ; \\
n=c+1, \ldots, k,
\end{gathered}
$$

where $q_{0}$ is obtained from the normalizing condition $\sum_{n=0}^{k} q_{n}=1$ and is given as

$$
\begin{aligned}
q_{0}= & {\left[\sum_{n=0}^{c} \lambda^{n} /\left\{n !(\mu+\nu)^{n}\right\}\right.} \\
& \left.+\sum_{n=c+1}^{k} \lambda^{n} \prod_{r=1}^{n-c}\{1-r /(k-c+1)\} /\left\{c !(\mu+\nu)^{c} \prod_{r=c+1}^{n}(c \mu+r \nu)\right\}\right]^{-1} .
\end{aligned}
$$

The steady state probabilities satisfy the recurrence relation, under R_EOS

$$
\begin{array}{ll}
q_{n}=[\lambda /\{n(\mu+\nu)\}] q_{n-1} ; & n=1,2, \ldots, c \\
q_{n}=\{\lambda(1-\overline{n-c / k-c+1}) /(c \mu+n \nu)\} q_{n-1} ; & n=c+1, \ldots, k .
\end{array}
$$

We shall denote by $S_{\mathrm{R} \_ \text {EOS }}$ the probability that an arriving unit has to wait on arrival (under R_EOS). Then

$$
S_{\text {R_EOS }}=\sum_{n=c}^{k} q_{n} .
$$




\section{Performance measures}

An important performance measure is ' $L$ ' which denotes the mean number of customers in the system. To obtain an expression for the same, we note that $L=P^{\prime}(1)$ where

$$
P^{\prime}(1)=\left.\frac{\mathrm{d}}{\mathrm{d} s} P(s)\right|_{s=1} .
$$

Here $P(S)$ is the p.g.f. of the steady state probabilities. The derivation of $P^{\prime}(1)$ is given in the appendix. From (A.5) and (8.2.4), the mean system size under two reneging rules are

$$
\begin{aligned}
L_{\mathrm{R} \_\mathrm{BOS}}= & {[1 /\{\lambda+\nu(k-c+1)\}]\left[\lambda k-c(\mu-\nu)(k-c+1) S_{\mathrm{R} \_\mathrm{BOS}}\right.} \\
& \left.+\{\lambda-(\mu-\nu)(k-c+1)\} M_{\mathrm{R} \_\mathrm{BOS}}+\lambda(c-1)\left(S_{\mathrm{R} \_\mathrm{BOS}}-1\right)\right]
\end{aligned}
$$

where

$$
M_{\mathrm{R} \_\mathrm{BOS}}=\sum_{n=1}^{c-1} n p_{n}
$$

and $S_{\mathrm{R} \_ \text {BOS }}$ is given in $(3.8)$.

$$
\begin{aligned}
L_{\mathrm{R} \_\mathrm{EOS}}= & {[1 /\{\lambda+\nu(k-c+1)\}]\left[\lambda k+\{\lambda-\mu(k-c+1)\} M_{\mathrm{R} \_\mathrm{EOS}}\right.} \\
& \left.\left.-c \mu(k-c+1) S_{\mathrm{R} \_ \text {EOS }}+\lambda(c-1)\right\}\left(S_{\mathrm{R} \_E O S}-1\right)\right]
\end{aligned}
$$

where

$$
M_{\mathrm{R} \_\mathrm{EOS}}=\sum_{n=1}^{c-1} n q_{n}
$$

and $S_{\text {R_EOS }}$ is given in (3.16).

Mean queue size can now be obtained and are given by

$$
\begin{aligned}
L_{q\left(\mathrm{R} \_\mathrm{BOS}\right)}= & \sum_{n=c+1}^{k}(n-c) p_{n} \\
= & L_{\mathrm{R} \_\mathrm{BOS}}-\sum_{n=1}^{c} n p_{n}-c+c \sum_{n=0}^{c} p_{n} \\
= & {[1 /\{\lambda+(k-c+1) \nu\}]\left[\lambda k-\{\lambda+\mu(k-c+1)\} c S_{\mathrm{R} \_\mathrm{BOS}}\right.} \\
& \left.-\mu(k-c+1) M_{\mathrm{R} \_\mathrm{BOS}}+\lambda(c-1)\left(S_{\mathrm{R} \_\mathrm{BOS}}-1\right)\right], \\
L_{q\left(\mathrm{R} \_\mathrm{EOS}\right)}= & \sum_{n=}^{k+1}(n-c) q_{n} \\
= & L_{\mathrm{R} \_\mathrm{EOS}}-\sum_{n=1}^{c} n q_{n}-c+c \sum_{n=0}^{c} q_{n} \\
= & {[1 /\{\lambda+(k-c+1) \nu\}]\left[\lambda k-(\mu+\nu)(k-c+1) M_{\mathrm{R} \_E O S}\right.} \\
& \left.-\{\lambda+(\mu+\nu)(k-c+1)\} c S_{\mathrm{R} \_\mathrm{EOS}}+\lambda(c-1)\left(S_{\mathrm{R} \_E O S}-1\right)\right] .
\end{aligned}
$$


Customers arrive into the system at the rate $\lambda$. However all the customers who arrive do not join the system because of balking or because of finite buffer restriction. The effective arrival rate into the system is thus different from the overall arrival rate and is given by

$$
\begin{aligned}
\lambda_{\left(\mathrm{R} \_\mathrm{BOS}\right)}^{e}= & \lambda \sum_{n=0}^{c-1} p_{n}+\lambda \sum_{n=c}^{k-1}\{1-(n-c+1) /(k-c+1)\} p_{n} \\
= & \lambda\left(1-S_{\mathrm{R} \_\mathrm{BOS}}\right)+\lambda\left(S_{\mathrm{R} \_\mathrm{BOS}}-p_{k}\right)-\{\lambda /(k-c+1)\} \\
& \times\left[L_{\mathrm{R} \_\mathrm{BOS}}-(k-c+1) p_{k}-M_{\mathrm{R} \_\mathrm{BOS}}-S_{\mathrm{R} \_\mathrm{BOS}}(c-1)\right] \\
= & \{\lambda /(k-c+1)\}\left[(k-c+1)-L_{\mathrm{R} \_\mathrm{BOS}}+M_{\mathrm{R} \_\mathrm{BOS}}+(c-1) S_{\mathrm{R} \_\mathrm{BOS}}\right] .
\end{aligned}
$$

Similarly in case of R_EOS

$$
\lambda_{\left(\mathrm{R} \_\mathrm{EOS}\right)}^{e}=\{\lambda /(k-c+1)\}\left[(k-c+1)-L_{\mathrm{R} \_\mathrm{EOS}}+M_{\mathrm{R} \_\mathrm{EOS}}+(c-1) S_{\mathrm{R} \_\mathrm{EOS}}\right] .
$$

We have assumed that each customer has a random patience time following $\exp (\nu)$. Clearly then, the reneging rate of the system would depend on the state of the system as well as the reneging rule. The average reneging rate under the two reneging rules are given by

$$
\begin{aligned}
& \operatorname{Avg} r r_{\left(\mathrm{R} \_\mathrm{BOS}\right)}=\sum_{n=c+1}^{k}(n-c) \nu p_{n} \\
& =\nu L_{q\left(\mathrm{R} \_\mathrm{BOS}\right)} \\
& =[\nu /\{\lambda+\nu(k-c+1)\}]\left[\lambda k-\mu(k-c+1) M_{\mathrm{R} \_\mathrm{BOS}}\right. \\
& \left.-\{\lambda+\mu(k-c+1)\} c S_{\mathrm{R} \_\mathrm{BOS}}+\lambda(c-1)\left(S_{\mathrm{R} \_\mathrm{BOS}}-1\right)\right] \text {. } \\
& \operatorname{Avg} r r_{(\text {R_EOS })}=\sum_{n=1}^{k} n \nu q_{n} \\
& =\nu L_{\mathrm{R} \_\mathrm{EOS}} \\
& =[\nu /\{\lambda+\nu(k-c+1)\}]\left[\lambda k-c \mu(k-c+1) S_{\text {R_EOS }}\right. \\
& \left.+\{\lambda-\mu(k-c+1)\} M_{\text {R_EOS }}+\lambda(c-1)\left(S_{\text {R_EOS }}-1\right)\right] .
\end{aligned}
$$

In a real life situation, customers who balk or renege represent the business lost. Customers are lost to the system in three ways, due to balking, due to reneging and due to finite buffer restriction. Management would like to know the proportion of total customers lost in order to have an idea of total business lost.

Hence the mean rate at which customers are lost (under R_BOS) is

$$
\begin{aligned}
\lambda & -\lambda_{\left(\mathrm{R} \_\mathrm{BOS}\right)}^{e}+\operatorname{avg} r r_{\left(\mathrm{R} \_\mathrm{BOS}\right)} \\
= & \{\lambda /(k-c+1)\}\left[L_{\mathrm{R} \_\mathrm{BOS}}-M_{\mathrm{R} \_\mathrm{BOS}}-(c-1) S_{\mathrm{R} \_\mathrm{BOS}}\right] \\
& +[\nu /\{\lambda+\nu(k-c+1)\}]\left[\lambda k-\mu(k-c+1) M_{\mathrm{R} \_\mathrm{BOS}}\right. \\
& \left.-\{\lambda+\mu(k-c+1)\} c S_{\mathrm{R} \_\mathrm{BOS}}+\lambda(c-1)\left(S_{\mathrm{R} \_\mathrm{BOS}}-1\right)\right],
\end{aligned}
$$


and the mean rate at which customers are lost (under R_EOS) is

$$
\begin{aligned}
\lambda & \left.-\lambda_{\left(\mathrm{R} \_E O S\right)}^{e}+\operatorname{avg} r r_{\left(\mathrm{R} \_E O S\right.}\right) \\
= & \{\lambda /(k-c+1)\}\left[L_{\mathrm{R} \_E O S}-M_{\mathrm{R} \_E O S}-(c-1) S_{\mathrm{R} \_E O S}\right] \\
& +[\nu /\{\lambda+\nu(k-c+1)\}]\left[\lambda k-c \mu(k-c+1) S_{\mathrm{R} \_E O S}\right. \\
& \left.\left.+\{\lambda-\mu(k-c+1)\} M_{\mathrm{R} \_E O S}+\lambda(c-1)\right\}\left(S_{\mathrm{R} \_E O S}-1\right)\right] .
\end{aligned}
$$

These rates helps in the determination of proportion of customers lost which is of interest to the system manager as also an important measure of business lost. This proportion (under R_BOS) is given by

$$
\begin{aligned}
\{\lambda & \left.-\lambda_{\left(\mathrm{R} \_\mathrm{BOS}\right)}^{e}+\operatorname{avg} r r_{\left(\mathrm{R} \_\mathrm{BOS}\right)}\right\} / \lambda \\
= & \{1 /(k-c+1)\}\left[L_{\mathrm{R} \_\mathrm{BOS}}-M_{\mathrm{R} \_\mathrm{BOS}}-(c-1) S_{\mathrm{R} \_\mathrm{BOS}}\right] \\
& +[\nu / \lambda\{\lambda+\nu(k-c+1)\}]\left[\lambda k-\mu(k-c+1) M_{\mathrm{R} \_\mathrm{BOS}}\right. \\
& \left.-\{\lambda+\mu(k-c+1)\} c S_{\mathrm{R} \_B O S}+\lambda(c-1)\left(S_{\mathrm{R} \_B O S}-1\right)\right],
\end{aligned}
$$

and the proportion (under R_EOS) is given by

$$
\begin{aligned}
& \left\{\lambda-\lambda_{(\text {R_EOS })}^{e}+\operatorname{avg} r r_{(\text {R_EOS })}\right\} / \lambda \\
& =\{1 /(k-c+1)\}\left[L_{\mathrm{R} \_\mathrm{EOS}}-M_{\mathrm{R} \_\mathrm{EOS}}-(c-1) S_{\mathrm{R} \_\mathrm{EOS}}\right] \\
& +[\nu / \lambda\{\lambda+\nu(k-c+1)\}]\left[\lambda k-c \mu(k-c+1) S_{\mathrm{R} \_E O S}\right. \\
& \left.\left.+\{\lambda-\mu(k-c+1)\} M_{\text {R_EOS }}+\lambda(c-1)\right\}\left(S_{\text {R_EOS }}-1\right)\right] \text {. }
\end{aligned}
$$

The proportion of customers completing receipt of service can now be easily determined from the above proportion.

The customers who leave the system from the queue do not receive service. Consequently, only those customers who reach the service station constitute the actual load of the server. From the server's point of view, this provides a measure of the amount of work he has to do. Let us call the rate at which customers reach the service station as $\lambda^{s}$. Then under R_BOS

$\lambda_{\left(\mathrm{R} \_\mathrm{BOS}\right)}^{s}=\lambda^{e} \quad$ (R_BOS) $(1-$ proportion of customers lost due to reneging out of those joining the system)

$$
\begin{aligned}
= & \lambda_{\left(\mathrm{R} \_\mathrm{BOS}\right)}^{e}\left\{1-\sum_{n=c+1}^{k}(n-k) \nu p_{n} / \lambda_{\left(\mathrm{R} \_\mathrm{BOS}\right)}^{e}\right\} \\
= & \lambda_{\left(\mathrm{R} \_\mathrm{BOS}\right)}^{e}-\operatorname{avg} r r_{\left(\mathrm{R} \_\mathrm{BOS}\right)} \\
= & \{\lambda /(k-c+1)\}\left\{(k-c+1)-L_{\mathrm{R} \_\mathrm{BOS}}+M_{\mathrm{R} \_\mathrm{BOS}}\right. \\
& \left.+(c-1) S_{\mathrm{R} \_\mathrm{BOS}}\right\}-[\nu /\{\lambda+\nu(k-c+1)\}]\left[\lambda k-\mu(k-c+1) M_{\mathrm{R} \_\mathrm{BOS}}\right. \\
& \left.-\{\lambda+\mu(k-c+1)\} c S_{\mathrm{R} \_\mathrm{BOS}}+\lambda(c-1)\left(S_{\mathrm{R} \_\mathrm{BOS}}-1\right)\right] .
\end{aligned}
$$

In case of R_EOS, one needs to recall that customers may renege even while being served and only those customers who renege from the queue will not constitute 
any work for the server. Then

$\lambda_{\left(\mathrm{R} \_\mathrm{EOS}\right)}^{s}=\lambda_{\left(\mathrm{R} \_\mathrm{EOS}\right)}^{e}$ (1-proportion of customers lost due to reneging from the queue out of those joining the system)

$$
\begin{aligned}
= & \lambda_{\left(\mathrm{R} \_\mathrm{EOS}\right)}^{e}\left\{1-\sum_{n=c+1}^{k}(n-k) \nu q_{n} / \lambda_{\left(\mathrm{R} \_\mathrm{EOS}\right)}^{e}\right\} \\
= & \lambda_{\left(\mathrm{R} \_\mathrm{EOS}\right)}^{e}-\nu\left\{L_{\mathrm{R} \_\mathrm{EOS}}-\sum_{n=1}^{c} n q_{n}\right\}+\nu c\left(1-\sum_{n=0}^{c} q_{n}\right) \\
= & \left.\lambda_{\left(\mathrm{R} \_\mathrm{EOS}\right)}^{e}-\nu L_{\mathrm{R} \_\mathrm{EOS}}+\nu M_{\mathrm{R} \_\mathrm{EOS}}+\nu c S_{\mathrm{R} \_\mathrm{EOS}}\right) \\
= & \{\lambda /(k-c+1)\}\left\{(k-c+1)-L_{\mathrm{R} \_\mathrm{EOS}}+M_{\mathrm{R} \_\mathrm{EOS}}\right. \\
& \left.+(c-1) S_{\mathrm{R} \_\mathrm{EOS}}\right\}-\nu L_{\mathrm{R} \_\mathrm{EOS}}+\nu\left(M_{\mathrm{R} \_\mathrm{EOS}}+c S_{\mathrm{R} \_\mathrm{EOS}}\right) .
\end{aligned}
$$

\section{SENSITIVITY ANALYSIS}

It is interesting to examine and understand how server utilization varies in response to change in system parameters. The system parameters of interest are $\lambda, \mu, \nu, k, c$ We place below the effect of change in these system parameters on server utilization. For this purpose, we shall follow the following convention in the rest of this section.

$p_{n}(\lambda, \mu, \nu, k, c)$ and $q_{n}(\lambda, \mu, \nu, k, c)$ will denote the probability that there are ' $n$ ' customers in a system with parameters $\lambda, \mu, \nu, k, c$ in steady state under R_BOS and R_EOS respectively.

It can be shown that:

(i) if $\lambda_{1}>\lambda_{0}$, then $\frac{p_{0}\left(\lambda_{1}, \mu, \nu, k, c\right)}{p_{0}\left(\lambda_{0}, \mu, \nu, k, c\right)}<1$.

This is because $p_{0}\left(\lambda_{1}, \mu, \nu, k, c\right)-p_{0}\left(\lambda_{0}, \mu, \nu, k, c\right)<0$

$$
\begin{aligned}
\Leftrightarrow & \frac{\left(\lambda_{0}-\lambda_{1}\right)}{\mu}+\ldots+\frac{\left(\lambda_{0}^{c}-\lambda_{1}^{c}\right)}{c ! \mu^{c}} \\
& +\frac{\{1-1 /(k-c+1)\}\left(\lambda_{0}^{c+1}-\lambda_{1}^{c+1}\right)}{c ! \mu^{c}(c \mu+\nu)}+\ldots \\
& +\frac{\{1-1 /(k-c+1)\} \ldots\{1-(k-c) /(k-c+1)\}\left(\lambda_{0}^{k}-\lambda_{1}^{k}\right)}{c ! \mu^{c}(c \mu+\nu) \ldots\{c \mu+(k-c) \nu\}}<0 .
\end{aligned}
$$

Hence $p_{0} \downarrow$ as $\lambda \uparrow$;

(ii) if $\mu_{1}>\mu_{0}$, then $\frac{p_{0}\left(\lambda, \mu_{1}, \nu, k, c\right)}{p_{0}\left(\lambda, \mu_{0}, \nu, k, c\right)}>1$. 
This is because $p_{0}\left(\lambda, \mu_{1}, \nu, k, c\right)-p_{0}\left(\lambda, \mu_{0}, \nu, k, c\right)>0$

$$
\begin{aligned}
\Leftrightarrow & \lambda\left(\frac{1}{\mu_{0}}-\frac{1}{\mu_{1}}\right)+\ldots+\frac{\lambda^{c}}{c !}\left(\frac{1}{\mu_{0}^{c}}-\frac{1}{\mu_{1}^{c}}\right) \\
& +\frac{\lambda^{c+1}}{c !}\left\{\frac{\{1-1 /(k-c+1)\}}{\mu_{0}^{c}\left(k \mu_{0}+\nu\right)}-\frac{\{1-1 /(k-c+1)\}}{\mu_{1}^{c}\left(k \mu_{1}+\nu\right)}\right\}+\ldots \\
+ & \frac{\lambda^{k}}{c !}\left\{\frac{\{1-1 /(k-c+1)\} \ldots\{1-(k-c) /(k-c+1)\}}{\mu_{0}^{c}\left(c \mu_{0}+\nu\right) \ldots\left\{c \mu_{0}+(k-c) \nu\right\}}\right. \\
& \left.-\frac{\{1-1 /(k-c+1)\} \ldots\{1-(k-c) /(k-c+1)\}}{\mu_{1}^{c}\left(c \mu_{1}+\nu\right) \ldots\left\{c \mu_{1}+(k-c) \nu\right\}}\right\}>0 .
\end{aligned}
$$

Hence $p_{0} \uparrow$ as $\mu \uparrow$;

(iii) if $\nu_{1}>\nu_{0}$, then $\frac{p_{0}\left(\lambda, \mu, \nu_{1}, k, c\right)}{p_{0}\left(\lambda, \mu, \nu_{0}, k, c\right)}>1$.

This is because $p_{0}\left(\lambda, \mu, \nu_{1}, k, c\right)-p_{0}\left(\lambda, \mu, \nu_{0}, k, c\right)>0$

$$
\begin{aligned}
\Leftrightarrow & \frac{\lambda^{\mathrm{c}+1}}{c ! \mu^{c}}\left\{\frac{\{1-1 /(k-c+1)\}}{\left(c \mu+\nu_{0}\right)}-\frac{\{1-1 /(k-c+1)\}}{\left(c \mu+\nu_{1}\right)}\right\} \\
& +\frac{\lambda^{k}}{c ! \mu^{c}}\left\{\frac{\{1-1 /(k-c+1)\} \ldots\{1-(k-c) /(k-c+1)\}}{\left(c \mu+\nu_{0}\right) \ldots\left\{c \mu+(k-c) \nu_{0}\right\}}\right. \\
& \left.-\frac{\{1-1 /(k-c+1)\} \ldots\{1-(k-c) /(k-c+1)\}}{\left(c \mu+\nu_{1}\right)\left\{c \mu+(k-c) \nu_{1}\right)}\right\}>0 .
\end{aligned}
$$

Hence $p_{0} \uparrow$ as $\nu \uparrow$;

(iv) if $k_{1}>k_{0}$ then $\frac{p_{0}\left(\lambda, \mu, \nu, k_{1}, c\right)}{p_{0}\left(\lambda, \mu, \nu, k_{0}, c\right)}<1$.

This is because $p_{0}\left(\lambda, \mu, \nu, k_{1}, c\right)-p_{0}\left(\lambda, \mu, \nu, k_{0}, c\right)<0$

$$
\Leftrightarrow \sum_{n=c+1}^{k_{0}} \frac{\lambda^{n} \prod_{r=1}^{n-c}\left\{1-r /\left(k_{0}-c+1\right)\right\}}{\prod_{r=1}^{n-c}(c \mu+r \nu)}-\sum_{n=c+1}^{k_{1}} \frac{\lambda^{n} \prod_{r=1}^{n-c}\left\{1-r /\left(k_{1}-c+1\right)\right\}}{\prod_{r=1}^{n-c}(c \mu+r \nu)}<0 .
$$

Hence $p_{0} \downarrow$ as $k \uparrow$.

The following can similarly be shown:

(v) $\quad q_{0} \downarrow$ as $\lambda \uparrow$;

(vi) $q_{0} \uparrow$ as $\mu \uparrow$;

(vii) $q_{0} \uparrow$ as $\nu \uparrow$;

(viii) $q_{0} \downarrow$ as $k \uparrow$; 
Under R_BOS, these results state that an increase in arrival rate would result in lowering of the fraction of time the server is idle. An increase in service rate would mean the server is able to work efficiently so that it can process same amount of work quickly. This translates to higher server idle time. An increase in reneging rate would mean the server has fewer work to do and hence higher fraction of idle time. An increase in system size translates to lowering of the fraction of time the server is idle. Similar conclusions can be drawn under R_EOS [18].

\section{NumeriCAL EXAMPLE}

To illustrate the use of our results, we apply them to a queuing problem. We quote below an example from Ravindran et al. [38], page 338.

"Consider a barbershop with two chairs, two barbers and no room for customers to wait. Say that the state of system is the number of customers in the shop: 0,1 or 2. If there is an empty chair when a customer arrives, he enters the shop and his haircut begins. If both chairs are occupied when he arrives, he does not enter the shop. As soon as a customer's haircut is completed, he leaves the shop instantaneously. The barbers do not assist one another when there is only one customer in the shop. On the average a customer arrives every $10 \mathrm{~min}$ and each haircut takes an average of $15 \mathrm{~min}$ ". Let us suppose that the barber shop is examining the implication of adding a few chairs for waiting customers. How many chairs should be added?

This is a design problem. Here $\lambda=6 / \mathrm{h}$ and $\mu=4 / \mathrm{h}$. Though not explicitly mentioned, it is necessary to assume reneging and balking. Let us consider a possible Markovian reneging rates of $\nu=1 / \mathrm{h}$. Given the fact that service in a barbershop is being analyzed, clearly the reneging rule would be R_BOS. We further assume that the probability of balking by an arriving customer is dependent on state of the system and is taken as ' $\{(n-c+1) /(k-c+1)\}$ ', $n=c, c+1, \ldots, k$ where $\mathrm{n}$ is the state of the customer observes the system to be in on its arrival.

Various performance measures of interest computed are given in Table 1. These measures were arrived at using a FORTRAN 77 program coded by the authors. Different choices of $k$ were considered (all rates in the table are per hour rates).

From Table 1 we can say that when there is no waiting space i.e. $k=2$ the proportion of customers lost is $31 \%$. If we add three more chairs, this proportion reduces to $20 \%$. The proportion of customers lost reduces by half $(1 / 2)$ if $k=11$.

\section{Conclusion}

The analysis of a multiserver Markovian finite buffer queuing system with statedependent balking and Markovian reneging has been presented. Though these concepts were discussed in literature, explicit expressions for $\mathrm{M} / \mathrm{M} / \mathrm{c} / \mathrm{K}$ system are not available. This paper makes a contribution in this direction. Closed form expressions of number of performance measures have been presented. To study the change in the system corresponding to change in system parameters sensitivity analysis 
TABle 1. Performance measures assuming $\lambda=6 / \mathrm{h}, \mu=4 / \mathrm{h}, c=2$ and $\nu=1 / \mathrm{h}$.

\begin{tabular}{|l|c|c|c|}
\hline \multirow{2}{*}{ Performance measure } & \multicolumn{3}{|c|}{ Number of chairs } \\
\cline { 2 - 4 } & $k=2$ & $k=5$ & $k=11$ \\
\hline Proportion of customers completing service & 0.68966 & 0.80052 & 0.84672 \\
\hline Fraction of time that all server is idle $\left(p_{0}\right)$ & 0.27586 & 0.22835 & 0.20855 \\
\hline Average length of system & 1.03448 & 1.42206 & 1.66539 \\
\hline Average length of queue & 0 & 0.22128 & 0.39531 \\
\hline Mean reneging rate & 0 & 0.22128 & 0.39531 \\
\hline Rate of loss due to balking and finite buffer. & 1.86207 & 0.97561 & 0.52437 \\
\hline Effective arrival rate & 4.13793 & 5.02439 & 5.47563 \\
\hline $\begin{array}{l}\text { Proportion of customers lost } \\
\text { (due to balking, reneging and finite buffer) }\end{array}$ & 0.31034 & 0.19948 & 0.15328 \\
\hline
\end{tabular}

has also been presented. A numerical example with design connotations has been presented to demonstrate results derived. The limitations of this work stem from the Markovian assumptions. Extension of our results for general distribution is a pointer to future research. We have assumed that the reneging distribution is position independent. In many scenarios, customers are aware of their position in the queue. This invariably causes waiting customers to have higher rates of reneging if their position in the queue is far away from the server compared to those who are near the server. This aspect has not been covered.

Acknowledgements. The authors wish to profusely thank the anonymous referee for his detailed, careful and exhaustive comments. These have led to very substantial improvement of the paper in the realm of conceptual exposition and presentation.

\section{ApPendix A}

\section{A.1. Derivation of $P^{\prime}(1)$ under R_BOS}

Let $P(s)$ denote the probability generating function, defined by $P(s)=\sum_{n=0}^{\infty} p_{n} s^{n}$.

From equation (3.2) we have

$$
\lambda p_{n-1}+(n+1) \mu p_{n+1}=\lambda p_{n}+n \mu p_{n} ; \quad n=1,2 \ldots, c-1 .
$$

Multiplying both sides of the equation by $s^{n}$ and summing over $n$

$$
\lambda s \sum_{n=1}^{k-1} p_{n-1} s^{n-1}-\lambda \sum_{n=1}^{k-1} p_{n} s^{n}=\sum_{n=1}^{k-1} \mu p_{n} s^{n}-\frac{1}{s} \mu \sum_{n=1}^{k-1}(n+1) p_{n+1} s^{n+1} .
$$


From (3.3) we have

$$
\begin{aligned}
& \lambda\{1-(n-c) /(k-c+1)\} p_{n-1}+\{c \mu+(n-c+1) \nu\} p_{n+1} \\
&=\lambda\{1-(n-c+1) /(k-c+1)\} p_{n}+\{c \mu+(n-c) \nu\} p_{n} \\
& n=c, c+1, \ldots, k-1 .
\end{aligned}
$$

Similarly multiplying both sides of the equation by $s^{n}$ and summing over $n$

$$
\begin{aligned}
\lambda s \sum_{n=c}^{k-1}\{1- & (n-c) /(k-c+1)\} p_{n-1} s^{n-1} \\
& -\lambda \sum_{n=c}^{k-1}\{1-(n-c+1) /(k-c+1)\} p_{n} s^{n} \\
= & \sum_{n=c}^{k-1}\{c \mu+(n-c) \nu\} p_{n} s^{n}-\frac{1}{s} \sum_{n=c}^{k-1}\{c \mu+(n-c+1) \nu\} p_{n+1} s^{n+1} .
\end{aligned}
$$

From (3.4) we have

$$
\lambda\{1-(k-c) /(k-c+1)\} p_{k-1}=\{c \mu+(k-c) \nu\} p_{k} .
$$

Multiplying both sides of the equation by $s^{k}$

$$
\lambda s\{1-(k-c) /(k-c+1)\} p_{k-1} s^{k-1}=\{c \mu+(k-c) \nu\} p_{k} s^{k} .
$$

Adding (A.1)-(A.3)

$$
\begin{aligned}
\Rightarrow & \lambda s\left[\sum_{n=1}^{c-1} p_{n-1} s^{n-1}+\sum_{n=c}^{k-1}\{1-(n-c) /(k-c+1)\} p_{n-1} s^{n-1}\right. \\
& \left.+\{1-(k-c) /(k-c+1)\} p_{k-1} s^{k-1}\right]-\lambda\left[\sum_{n=1}^{c-1} p_{n} s^{n}\right. \\
& \left.+\sum_{n=c}^{k-1}\{1-(n-c+1) /(k-c+1)\} p_{n} s^{n}\right] \\
= & \mu s \sum_{n=1}^{c-1} n p_{n} s^{n-1}+\sum_{n=c}^{k-1}\{c \mu+(n-c) \nu\} p_{n} s^{n}+\left\{c \mu+(k-c) p_{k} s^{k}\right\} \\
& -\frac{1}{s}\left[\mu \sum_{n=1}^{c-1}(n+1) p_{n+1} s^{n+1}+\sum_{n=c}^{k-1}\{c \mu+(n-c+1) \nu\} p_{n+1} s^{n+1}\right]
\end{aligned}
$$




$$
\begin{aligned}
& \Rightarrow \lambda s\left[p_{0} s^{0}+p_{1} s^{1}+\ldots+p_{c-1} s^{c-1}+\{1-1 /(k-c+1)\} p_{c} s^{c}+\ldots\right. \\
& \left.+\{1-(k-c) /(k-c+1)\} p_{k-1} s^{k-1}\right] \\
& -\lambda\left[p_{1} s^{1}+\ldots+p_{c-1} s^{c-1}+\{1-1 /(k-c+1)\} p_{c} s^{c}+\ldots\right. \\
& \left.+\{1-(k-c) /(k-c+1)\} p_{k-1} s^{k-1}\right] \\
& =\mu s\left\{P^{\prime}(s)-\sum_{n=c}^{k} n p_{n} s^{n-1}\right\}+c \mu\left\{P(s)-\sum_{n=0}^{c-1} p_{n} s^{n}\right\} \\
& +\nu s\left\{(c+1) p_{c+1} s^{c}+\ldots+k p_{k} s^{k-1}\right\}-\nu c\left\{p_{c+1} s^{c+1}+\ldots+p_{k} s^{k}\right\} \\
& \frac{1}{s}\left[\mu s\left\{P^{\prime}(s)-p_{1}-\sum_{n=c+1}^{k} n p_{n} s^{n-1}\right\}+c \mu\left\{P(s)-\sum_{n=0}^{c-1} p_{n} s^{n}\right\}\right. \\
& \left.+\nu s\left\{(c+1) p_{c+1} s^{c}+\ldots+k p_{k} s^{k-1}\right\}-\nu c\left\{p_{c+1} s^{c+1}+\ldots+p_{k} s^{k}\right\}\right] \\
& \Rightarrow \lambda s\left[\left\{P(s)-p_{k} s^{k}\right\}-\{s /(k-c+1)\}\left\{c p_{c} s^{c-1}+\ldots+(k-1) p_{k-1} s^{k-2}\right\}\right. \\
& \left.+\{(c-1) /(k-c+1)\}\left\{p_{c} s^{c}+\ldots+p_{k-1} s^{k-1}\right\}\right] \\
& -\lambda\left[\left\{P(s)-p_{0}-p_{k} s^{k}\right\}-\{s /(k-c+1)\}\left\{c p_{c} s^{c-1}+\ldots+(k-1) p_{k-1} s^{k-2}\right\}\right. \\
& \left.+\{(c-1) /(k-c+1)\}\left\{p_{c} s^{c}+\ldots+p_{k-1} s^{k-1}\right\}\right] \\
& =\mu s\left\{P^{\prime}(s)-\sum_{n=c}^{k} n p_{n} s^{n-1}\right\}+c \mu\left\{P(s)-\sum_{n=0}^{c-1} p_{n} s^{n}\right\} \\
& +\nu s\left\{P^{\prime}(s)-\sum_{n=1}^{c} n p_{n} s^{n-1}\right\}-c \nu\left\{P(s)-\sum_{n=0}^{c} p_{n} s^{n}\right\} \\
& -\mu\left\{P^{\prime}(s)-p_{1}-\sum_{n=c+1}^{k} n p_{n} s^{n-1}\right\}-\frac{c \mu}{s}\left\{P(s)-\sum_{n=0}^{c-1} p_{n} s^{n}\right\} \\
& -\nu\left\{P^{\prime}(s)-\sum_{n=1}^{c} n p_{n} s^{n-1}\right\}+\frac{c \nu}{s}\left\{P(s)-\sum_{n=0}^{c} p_{n} s^{n}\right\} \\
& \Rightarrow \lambda s\left\{P(s)-p_{k} s^{k}\right\}-\left\{\lambda s^{2} /(k-c+1)\right\}\left\{P^{\prime}(s)-k p_{k} s^{k-1}\right.
\end{aligned}
$$$$
\left.-\sum_{n=1}^{c-1} n p_{n} s^{n-1}\right\}+\{\lambda s(c-1) /(k-c+1)\}\left\{P(s)-p_{k} s^{k}-\sum_{n=0}^{c-1} p_{n} s^{n}\right\}
$$$$
-\lambda\left\{P(s)-p_{0}-p_{k} s^{k}\right\}+\{\lambda s /(k-c+1)\}\left\{P^{\prime}(s)-k p_{k} s^{k-1}\right.
$$$$
\left.-\sum_{n=1}^{c-1} n p_{n} s^{n-1}\right\}-\{\lambda(c-1) /(k-c+1)\}\left\{P(s)-p_{k} s^{k}-\sum_{n=0}^{c-1} p_{n} s^{n}\right\}
$$$$
=\mu s\left\{P^{\prime}(s)-\sum_{n=c}^{k} n p_{n} s^{n-1}\right\}+c \mu\left\{P(s)-\sum_{n=0}^{c-1} p_{n} s^{n}\right\}
$$$$
+\nu s\left\{P^{\prime}(s)-\sum_{n=1}^{c} n p_{n} s^{n-1}\right\}-c \nu\left\{P(s)-\sum_{n=0}^{c} p_{n} s^{n}\right\}
$$ 


$$
\begin{aligned}
& -\mu\left\{P^{\prime}(s)-p_{1}-\sum_{n=c+1}^{k} n p_{n} s^{n-1}\right\}-\frac{c \mu}{s}\left\{P(s)-\sum_{n=0}^{c-1} p_{n} s^{n}\right\} \\
& -\nu\left\{P^{\prime}(s)-\sum_{n=1}^{c} n p_{n} s^{n-1}\right\}+\frac{c \nu}{s}\left\{P(s)-\sum_{n=0}^{c} p_{n} s^{n}\right\} \\
& \Rightarrow\left\{\lambda s P^{\prime}(s)(1-s)\right\} /(k-c+1)+\mu P^{\prime}(s)(1-s)+\nu P^{\prime}(s)(1-s) \\
& =-c \mu P(s)\{(1 / s)-1\}+c \nu P(s)\{(1 / s)-1\}+\lambda P(s)(1-s) \\
& +\{\lambda(c-1) /(k-c+1)\} P(s)(1-s)+\lambda s p_{k} s^{k}-\lambda s p_{k} s^{k} \\
& +\{\lambda s(1-s) /(k-c+1)\} \sum_{n=1}^{c-1} n p_{n} s^{n-1}-\lambda p_{k} s^{k}+\lambda p_{k} s^{k} \\
& -\{\lambda(c-1)(1-s) /(k-c+1)\} \sum_{n=0}^{c-1} p_{n} s^{n}+\mu(1-s) \\
& \times \sum_{n=c}^{k} n p_{n} s^{n-1}+c \mu\{(1 / s)-1\} \\
& \times \sum_{n=0}^{c} p_{n} s^{n}+\nu(1-s) \sum_{n=1}^{c} n p_{n} s^{n-1}-\nu c\{(1 / s)-1\} \\
& \times \sum_{n=0}^{c} p_{n} s^{n}-c \mu p_{c} s^{c}\{(1 / s)-1\} \\
& \Rightarrow P^{\prime}(s)[\{\lambda s+(\mu+\nu)(k-c+1)\} /(k-c+1)]=[\{\lambda s(c-1)+\lambda s(k-c+1) \\
& -c(\mu-\nu)(k-c+1)\} /(k-c+1) s] P(s) \\
& +\{\lambda s /(k-c+1)\} \sum_{n=0}^{c-1} n p_{n} s^{n-1} \\
& -\{\lambda(c-1) /(k-c+1)\} \sum_{n=0}^{c-1} p_{n} s^{n}+\mu \sum_{n=c}^{k} n p_{n} s^{n-1} \\
& +(c \mu / s) \sum_{n=0}^{c-1} p_{n} s^{n}+\nu \sum_{n=1}^{c} n p_{n} s^{n-1}-(c \nu / s) \sum_{n=0}^{c} p_{n} s^{n}
\end{aligned}
$$

Now

$$
\begin{aligned}
\Rightarrow & \lim _{s \rightarrow 1-} P^{\prime}(s)[\{\lambda s+(\mu+\nu)(k-c+1)\} /(k-c+1)] \\
= & \lim _{s \rightarrow 1-}[\{\lambda s(c-1)+\lambda s(k-c+1)-c(\mu-\nu)(k-c+1)\} /(k-c+1) s] P(s) \\
& +\{\lambda s /(k-c+1)\} \sum_{n=0}^{c-1} n p_{n} s^{n-1}-\{\lambda(c-1) /(k-c+1)\} \sum_{n=0}^{c-1} p_{n} s^{n} \\
& +\mu \sum_{n=c}^{k} n p_{n} s^{n-1}+(c \mu / s) \sum_{n=0}^{c-1} p_{n} s^{n}+\nu \sum_{n=1}^{c} n p_{n} s^{n-1}-(c \nu / s) \sum_{n=0}^{c} p_{n} s^{n}
\end{aligned}
$$




$$
\begin{aligned}
\Rightarrow & P^{\prime}(1)[\{\lambda+(\mu+\nu)(k-c+1)\} /(k-c+1)] \\
= & {[\{\lambda k-c(\mu-\nu)(k-c+1)\} /(k-c+1)] } \\
& +\{\lambda /(k-c+1)\} \sum_{n=0}^{c-1} n p_{n}-\{\lambda(c-1) /(k-c+1)\} \sum_{n=0}^{c-1} p_{n} \\
& +\mu \sum_{n=c}^{k} n p_{n}+c \mu \sum_{n=0}^{c-1} p_{n}+\nu \sum_{n=1}^{c} n p_{n}-c \nu \sum_{n=0}^{c} p_{n} \\
\Rightarrow & P^{\prime}(1)=[1 /\{\lambda+(\mu+\nu)(k-c+1)\}][\{\lambda-c(\mu-\nu)(k-c+1)\} \\
& +\lambda \sum_{n=0}^{c-1} n p_{n}-\lambda(c-1) \sum_{n=0}^{c-1} p_{n}+\mu(k-c+1)\left\{P^{\prime}(1)-\sum_{n=0}^{c-1} n p_{n}\right\} \\
& \left.+c \mu(k-c+1) \sum_{n=0}^{c-1} p_{n}+\nu(k-c+1) \sum_{n=1}^{c} n p_{n}-c \nu(k-c+1) \sum_{n=0} p_{n}\right] \\
\Rightarrow & P^{\prime}(1)=[1 /\{\lambda+\nu(k-c+1)\}][\{\lambda-c(\mu-\nu)(k-c+1)\} \\
& +\{\lambda-\mu(k-c+1)\} \sum_{n=0}^{c-1} n p_{n}+\{c \mu(k-c+1)-\lambda(c-1)\} \sum_{n=0}^{c-1} p_{n} \\
& \left.+\nu(k-c+1) \sum_{n=1}^{c} n p_{n}-c \nu(k-c+1) \sum_{n=0}^{c} p_{n}\right] .
\end{aligned}
$$

Using (3.8) and (4.2) in (8.1.4) we get

$$
\begin{aligned}
\Rightarrow & P^{\prime}(1)=[1 /\{\lambda+\nu(k-c+1)\}]\left[\{\lambda k-c(\mu-\nu)(k-c+1)\} S_{\mathrm{R} \_\mathrm{BOS}}\right. \\
& \left.\left.+\{\lambda-(\mu-\nu)(k-c+1)\} M_{\mathrm{R} \_\mathrm{BOS}}+\lambda(c-1)\right\}\left(S_{\mathrm{R} \_\mathrm{BOS}}-1\right)\right] .
\end{aligned}
$$

\section{A.2 Derivation of Q'(1) Under R_EOS}

From equation (3.10) we have,

$$
\lambda q_{n-1}+(n+1)(\mu+\nu) q_{n+1}=\lambda q_{n}+n(\mu+\nu) q_{n} ; \quad n=1,2, \ldots, c-1 .
$$

Multiplying both sides of this equation by $s^{n}$ and summing over $n$ from we get

$$
\lambda s \sum_{n=1}^{c-1} q_{n-1} s^{n-1}-\lambda \sum_{n=1}^{c-1} q_{n} s^{n}=(\mu+\nu) \sum_{n=1}^{c-1} n q_{n} s^{n}-\frac{1}{s}(\mu+\nu) \sum_{n=1}^{c-1}(n+1) q_{n+1} s^{n+1} .
$$

From equation (3.11)

$$
\begin{aligned}
\lambda\{1-(n-c) /(k-c+1)\} q_{n-1}+\{c \mu+(n+1) \nu\} q_{n+1} & \\
=\lambda\{1-(n-c+1) /(k-c+1)\} q_{n}+(c \mu+n \nu) q_{n} & \\
& n=c, c+1, \ldots, k-1 .
\end{aligned}
$$


Multiplying both sides of this equation by $s^{n}$ and summing over $n$ from we get

$$
\begin{aligned}
\lambda s \sum_{n=c}^{k-1}\{1-(n-c) / & (k-c+1)\} q_{n-1} s^{n-1}-\lambda \sum_{n=c}^{k-1}\{1-(n-c+1) /(k-c+1)\} q_{n} s^{n} \\
= & \left.\sum_{n=c}^{k-1}\{c \mu+n \nu\} q_{n} s^{n}-\frac{1}{s} \sum_{n=c}^{k-1}\{c \mu+(n+1) \nu\} q_{n+1} s^{n+1} . \quad \text { (A. } 7\right)
\end{aligned}
$$

From (3.12) we have

$$
\lambda\{1-(k-c) /(k-c+1)\} q_{k-1}=(c \mu+k \nu) q_{k} .
$$

Multiplying both sides of the equation by $s^{k}$

$$
\lambda s\{1-(k-c) /(k-c+1)\} q_{k-1} s^{k-1}=(c \mu+k \nu) q_{k} s^{k} .
$$

Adding (A.6), (A.7) and (A.8) and proceeding in a manner similar to Section A.1, we obtain,

$$
\begin{aligned}
\Rightarrow & Q^{\prime}(1)=[1 /\{\lambda+\nu(k-c+1)\}]\left[\left\{\lambda k+\{\lambda-\mu(k-c+1)\} M_{\text {R_EOS }}\right.\right. \\
& \left.\left.-c \mu(k-c+1) S_{\text {R_EOS }}+\lambda(c-1)\right\}\left(S_{\text {R_BOS }}-1\right)\right] .
\end{aligned}
$$

\section{REFERENCES}

[1] E. Altman and U. Yechiali, Analysis of customer impatience in queue with server vacations. Queuing Syst. 52 (2006) 261-279.

[2] E. Altman and U. Yechiali, Infinite-server queues with system's additional tasks and impatient customers. Probab. Eng. Inform. Sci. 22 (2008) 477-493.

[3] R.O. Al-Seedy and F.M. Al-Ibraheem, An interarrival hyperexponential machine interference with balking, Reneging, state-dependent, spares and an additional server for longer queues. Int. J. Math. \& Math. Sci. 27 (2001) 737-747.

[4] R.O. Al-Seedy, A.A. El-Sherbiny, S.A. El-Shehawy and S.I. Ammar, Transient solution of the M/M/C queue with balking and reneging. Comput. Math. Appl. 57 (2009) 1280-1285.

[5] C.J. Ancker Jr. and A.V. Gafarian, Queuing problems with balking and reneging I. Oper. Res. 11 (1963) 88-100.

[6] J. Bae and S. Kim, The stationary workload of the G/M/1 queue with impatient customers. Queuing Syst. 64 (2010) 253-265.

[7] J. Bae, S. Kim and E.Y. Lee, The virtual waiting time of the M/G/1 queue with impatient customers. Queuing Syst. 38 (2001) 485-494.

[8] F. Baccelli, P. Boyer and G. Hebuterne, Single server queues with impatients customers. Adv. Appl. Probab. 16 (1984) 887-905.

[9] D.Y. Barrer, Queuing with impatient customers and ordered service. Oper. Res. 5 (1957) 650-656.

[10] D.Y. Barrer, Queuing with impatient customers and indifferent clerks. Oper. Res. 5 (1957) 644-649.

[11] N.K. Boots and H. Tijms, An M/M/c queues with impatient customers. TOP 7 (1999) $213-220$.

[12] O. Boxma, D. Perry, W. Stadje and S. Zacks, The busy period of an M/G/1 queue with customer impatience. J. Appl. Probab. 47 (2010) 130-145. 
[13] A. Brandt and M. Brandt, On a two-queue priority system with impatience and its application to a call centre. Methodol. Comput. Appl. Probab. 1 (1999) 191-210.

[14] B.D. Choi, B. Kim and J. Chung, M/M/1 queue with impatient customers of higher priority. Queuing Syst. 38 (2001) 49-66.

[15] B.D. Choi, B. Kim and D. Zhu, MAP/M/C queue with constant impatients times. Math. Oper. Res. 29 (2004) 309-325.

[16] A. Choudhury, Impatience in single server queuing model. Am. J. Math. Manag. Sci. 28 (2008) 177-211.

[17] A. Choudhury and P. Medhi, Balking and reneging in multiserver Markovian queuing system. Int. J. Math. Oper. Res. 3 (2011) 377-394.

[18] A. Choudhury and P. Medhi, Some aspects of balking and reneging in finite buffer queues. RAIRO Oper. Res. 45 (2011) 223-240.

[19] J.K. Cochran and J.R. Broyles, Developing non-linear queuing regressions to increase emergency department patient safety: approximating reneging with balking. Comput. Ind. Eng. 59 (2010) 378-386.

[20] M.S. El-Paoumy, On Poisson arrival queue: $\mathrm{M}^{\mathrm{X}} / \mathrm{M} / 2 / \mathrm{N}$ with balking, reneging and heterogeneous servers. Appl. Math. Sci. 2 (2008) 1169-1175.

[21] M.S. El-Paoumy, On a truncated Erlangian queuing system with state-dependent service rate, balking and reneging. Appl. Math. Sci. 2 (2008) 1161-1167.

[22] M.S. El-Paoumy and M.M. Ismail, On a truncated erlang queuing system with bulk arrivals, balking and reneging. Appl. Math. Sci. 3 (2009) 1103-1113.

[23] A.A. El-Sherbiny, The non-truncated bulk arrival queue $\mathrm{M}^{\mathrm{X}} / \mathrm{M} / 1$ with reneging, balking, state-dependent and an additional server for longer queues. Appl. Math. Sci. 2 (2008) 747752 .

[24] B. Gavish and P.J. Schweitzer, The Markovian queue with bounded waiting time. Manage. Sci. 23 (1977) 1349-1357.

[25] A. Ghosal, Queues with finite waiting time. Oper. Res. 11 (1963) 919-921.

[26] A.M. Haghighi, J. Medhi and S.G. Mohanty, On a multi server Markovian queuing system with balking and reneging. Comput. Oper. Res. 13 (1986) 421-425.

[27] F.A. Haight, Queuing with balking. Biometrika 44 (1957) 360-369.

[28] F.A. Haight, Queuing with reneging. Metrika 2 (1959) 186-197.

[29] O. Jouini, Z. Aksin and Y. Dallery, Call centers with delay information: models and insights (2008). Downloaded from the site www.uclouvain.be/cps/ucl/doc/core/documents/ Jouini.pdf (accessed on 03.08.2010).

[30] O. Jouini, Y. Dallery and Z. Aksin, Queuing models for full-flexible multi-class call centers with real-time anticipated delays. Int. J. Prod. Econ. 120 (2009) 389-399.

[31] J.C. Ke and K.-H. Wang, Cost analysis of the M/M/R machine repair problem with balking, reneging and server breakdowns. J. Oper. Res. Soc. 50 (1999) 275-282.

[32] A.G.D. Kok and H. Tijms, A queuing system with impatient customers. J. Appl. Probab. 22 (1985) 688-696.

[33] L. Liu and V.G. Kulkarni, Busy period analysis for M/PH/1 queues with workload dependent balking. Queuing Syst. 59 (2008) 37-51.

[34] L. Liu and V.G. Kulkarni, Balking and reneging in M/G/s system exact analysis and approximations. Probab. Eng. Inform. Sci. 22 (2008) 355-371.

[35] L. Liu, B.R.K. Kashyap and J.G.C. Templeton, The service system $M / M^{R} / \infty$ with impatient customers. Queuing Syst. 2 (1987) 363-372.

[36] M. Martin and J.R. Artalejo, Analysis of an M/G/1 Queue with two types of Impatient units. Adv. Appl. Probab. 27 (1995) 840-861.

[37] A. Pazgal and S. Radas, Comparison of customer balking and reneging behavior to queuing theory predictions: an experimental study. Comput. Oper. Res. 35 (2008) 2537-2548.

[38] A. Ravindran, D.T. Phillips and J.J. Solberg, Operations research, principles and practice, 2nd edition. John Wiley and Sons, New York (1987).

[39] A.I. Shawky, The single server machine interference model with balking, reneging and an additional server for longer queues. Microelectron. Reliab. 37 (1997) 355-357. 
[40] A.I. Shawky and M.S. El-Paoumy, The interarrival hyperexponential queues: $\mathrm{H}_{\mathrm{k}} / \mathrm{M} / \mathrm{c} / \mathrm{N}$ with balking and reneging. Stochastics An Int. J. of Probability \& Stochastic Processes 69 (2000) 67-76.

[41] A.I. Shawky and M.S. El-Paoumy, The truncated hyper-poisson queues: $\mathrm{H}_{\mathrm{k}} / \mathrm{M}^{\mathrm{a}, \mathrm{b}} / \mathrm{c} / \mathrm{N}$ with balking, reneging and general bulk-service rule. Yugosl. J. Oper. Res. 8 (2008) 23-36.

[42] C.J. Singh, B. Kumar and M. Jain, Single server interdependent queuing model with controllable arrival rates and reneging. Pakistan J. Stat. 23 (2007) 171-178.

[43] W. Xiong and T. Altiok, An approximation for multi-server queues with deterministic reneging times. Ann. Oper. Res. 172 (2009) 143-151.

[44] W. Xiong, D. Jagerman and T. Altiok, M/G/1 queue with deterministic reneging times. Perform. Eval. 65 (2008) 308-316.

[45] U. Yechiali, Queues with system disasters and impatient customers when system is down. Queueing Syst. 56 (2007) 195-202.

[46] D. Yue and W. Yue, A heterogeneous two-server network system with balking and a Bernoulli vacation schedule. J. Ind. Manag. Optim. 6 (2010) 501-516.

[47] D. Yue, W. Yue, J. Yu and R. Tian, A heterogeneous two-server queuing system with balking and server breakdowns. Paper presented in the Eight International Symposium on Operations Research and its Applications (ISORA'09). Zhangjiajie, Chaina (2009). Downloaded from the site www. aporc.org/LNOR/10/ISORA2009F31.pdf on 04.08.2010.

[48] Y. Zhang, D. Yue and W. Yue, Analysis of an M/M/1/N queue with balking, reneging and server vacations. Paper presented in the Vth. International Symposium on OR and its Application (2005). Downloaded from www.aporc.org/LNOR/6/ISORA2006F10.pdf on 04.08.2010. 